\title{
Top-down guidance in visual search for facial expressions
}

\author{
Sowon Hahn ANd Scott D. Gronlund \\ University of Oklahoma, Norman, Oklahoma
}

\begin{abstract}
Using a visual search paradigm, we investigated how a top-down goal modified attentional bias for threatening facial expressions. In two experiments, participants searched for a facial expression either based on stimulus characteristics or a top-down goal. In Experiment 1, participants searched for a discrepant facial expression in a homogenous crowd of faces. Consistent with previous research, we obtained a shallower response time (RT) slope when the target face was angry than when it was happy. In Experiment 2, participants searched for a specific type of facial expression (allowing a top-down goal). When the display included a target, we found a shallower RT slope for the angry than for the happy face search. However, when an angry or happy face was present in the display in opposition to the task goal, we obtained equivalent RT slopes, suggesting that the mere presence of an angry face in opposition to the task goal did not support the well-known angry face superiority effect. Furthermore, RT distribution analyses supported the special status of an angry face only when it was combined with the top-down goal. On the basis of these results, we suggest that a threatening facial expression may guide attention as a high-priority stimulus in the absence of a specific goal; however, in the presence of a specific goal, the efficiency of facial expression search is dependent on the combined influence of a top-down goal and the stimulus characteristics.
\end{abstract}

Many studies indicate that negative or threat-related facial expressions guide attention more efficiently than neutral or happy facial expressions (Eastwood, Smilek, \& Merikle, 2001; Fox et al., 2000; Hansen \& Hansen, 1988; Öhman, Lundqvist, \& Esteves, 2001; Vuilleumier $\&$ Schwartz, 2001). The special status of threatening facial expressions in guiding attention has been observed even when the face was presented outside an individual's awareness (Dimberg, Thunberg, \& Elmehed, 2000; Mogg \& Bradley, 1999; Morris, Öhman, \& Dolan, 1998). Neuropsychological studies have suggested that threatening facial expressions are processed through thalamic pathways to the amygdala, which allows an automatic response to a potential threat before cortical processing is complete (LeDoux, 1996; Öhman, 2002).

The purpose of the present study was to investigate whether a voluntary search goal would modify a seemingly automatic attentional bias to threatening facial expressions. In the human visual environment, faces are undeniably special, but the importance of facial expressions may depend on the goal at hand. If immediate survival is at stake, attentional alert for potential threat is essential. However, if a person has other goals, such as emotional regulation, blind alert to a threatening facial expression may interfere with the current goal. Therefore, it is conceivable that a top-down goal would modify attentional bias to threatening facial expressions. Research has also found that the bias toward the angry face can be modified. For instance, anxious individuals have been found to be more biased to threatening faces than less-anxious individuals (Fox, Russo, Bowles, \& Dutton, 2001), older adults have been found to be better at inhibiting angry faces than younger adults (Hahn, Carlson, Singer, \& Gronlund, 2006; Mather \& Carstensen, 2003), and task instruction has been found to modify visual search advantage for threatening and nonthreatening facial expression search (Williams, Moss, Bradshaw, \& Mattingley, 2005).

To investigate how a top-down search goal would influence attentional guidance for facial expressions, we conducted two experiments in which participants searched for a facial expression either on the basis of stimulus characteristics or a top-down goal. In Experiment 1, participants searched for a discrepant facial expression in a crowd of homogenous faces. In Experiment 2, participants searched for a specific target, either a happy face or an angry face (allowing a top-down goal). Given the special status of an angry face in guiding attention, we hypothesized that without a top-down goal, the angry face would guide more effective visual search than the happy face. We also hypothesized that a top-down goal might either override or compete with a stimulus-based search. Therefore, we predicted that the angry face superiority effect and the topdown goal would show a combined influence on search.

\section{EXPERIMENT 1 Discrepant Facial Expression Search}

In Experiment 1, participants viewed a number of schematic faces on a computer screen and determined the presence or absence of a discrepant facial expression in a

S.Hahn, sowon@ou.edu 
crowd of neutral faces. We measured response time (RT) and accuracy data to examine search effectiveness. On the basis of the previous research, we expected that an angry face, as a biologically significant stimulus, would guide attention more effectively (Eastwood et al., 2001; Fox et al., 2000; Hansen \& Hansen, 1988).

\section{Method}

Participants. Sixteen young adults (mean age $=21.1$ years; 5 males, 11 females) participated in exchange for class credit.

Stimuli and Apparatus. A Dell 8100 computer with 3-GHz Pentium 4 processors and a 17 -in. LCD monitor was used for stimuli presentation and response input. The participants responded by pressing the $\mathrm{K}$ or $\mathrm{F}$ key on the computer keyboard. Each trial display consisted of a number of schematic faces on the screen. There were $5,10,15$, or 20 faces in each display. Each face was approximately $1.5 \mathrm{~cm} \times 1.5 \mathrm{~cm}$ in width and height. Examples of the stimulus display are illustrated in Figure 1.

Procedure. Each session started with an instruction screen. When the participants were ready, they pressed the space bar to begin. The stimulus display remained on the screen until participants pressed the $\mathrm{K}$ or $\mathrm{F}$ key to indicate either the presence or absence of a discrepant facial expression. The keys were counterbalanced over participants. After the participants responded by pressing a key, a blank screen appeared for $200 \mathrm{msec}$, followed by the next trial display. A total of 600 trials were included in the experimental session. For one third of the trials, the display consisted of only neutral faces (targetabsent trials). Another one third of the trials included one happy face and the rest neutral faces. The remaining one third of the trials included one angry face and the rest neutral faces.

\section{Results}

The mean RTs and accuracy for each of the conditions are presented in Figure 2. We submitted the mean RT and accuracy data to two-way ANOVAs, with the discrepant face type (absent, happy, angry) and set size $(5,10,15,20)$ as within-subjects variables. The partial eta squared $\left(\eta_{\mathrm{p}}^{2}\right)$ representing the proportion of variance explained in the dependent variable is reported for each significant effect.

For the RT analysis, main effects were obtained for the discrepant face type $\left[F(2,30)=70.1, p<.0001, \eta_{\mathrm{p}}^{2}=.82\right]$ and set size $\left[F(3,45)=55.5, p<.0001, \eta_{\mathrm{p}}^{2}=.79\right]$, indicating that an angry face was detected faster than a happy face, and that RT increased with increasing set size. We also found a significant interaction for discrepant face type $\times$ set size $\left[F(6,90)=31.6, p<.0001, \eta_{\mathrm{p}}^{2}=.68\right]$, indicating that the RT slope increased as set size increased. The average increase of RT over set size resulted in slopes of 112, 52, and $37 \mathrm{msec}$ for the target-absent, happy discrepant face, and angry discrepant face trials, respectively. Post hoc comparison with Duncan tests $(\alpha=.05)$ showed that all three slopes were different from each other. For the accuracy data, we found a significant interaction for discrepant face type $\times$ set size $\left[F(6,90)=2.5, p<.03, \eta_{\mathrm{p}}^{2}=.14\right]$, which indicated that the accuracy for happy discrepant decreased with increasing set size, but accuracy for angry discrepant remained constant. None of the other effects were significant.

\section{Discussion}

Consistent with the angry face superiority effect in visual search (Eastwood et al., 2001; Fox et al., 2000;
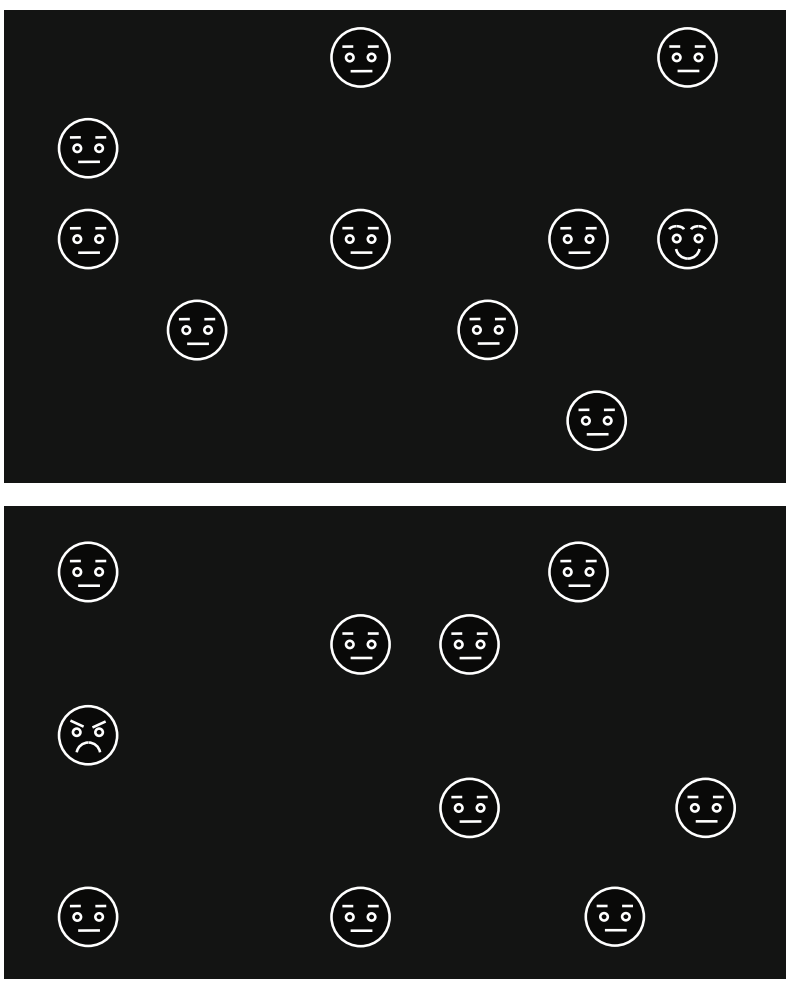

Figure 1. Examples of the displays used in Experiment 1. The upper panel shows a happy discrepant face trial and the lower panel shows an angry discrepant face trial.

Hansen \& Hansen, 1988; Öhman et al., 2001), we found a smaller search slope in the angry discrepant face condition than in the happy discrepant face condition. In the present study, the target type was not explicitly specified; thus, the smaller search slope for an angry face target indicated that angry faces attract attention more effectively than happy faces.

From an evolutionary perspective, threat processing should be automatic, perhaps due to a special brain module dedicated as a threat-alert mechanism. Researchers have suggested that facial expressions are processed through a "quick and dirty" analysis via a simple subcortical network accessing the amygdala prior to complete cortical visual processing (LeDoux, 1996; Öhman, 2002). However, previous visual search studies have not found evidence of automatic attention capture by threatening facial expressions. Instead, studies have supported that angry facial expressions guide more effective search (Eastwood et al., 2001; Fox et al., 2000; Nothdurft, 1993).

We hypothesized that the angry face superiority effect in visual search may be modulated by a differential influence of top-down and stimulus-based priority. Without a specific top-down goal, search efficiency may be influenced by biologically significant stimuli (i.e., threatening facial expressions). However, a top-down goal might modify the priority structure of the task. In Experiment 2, we investigated the role of a top-down goal on the attentional priorities of facial expressions. 


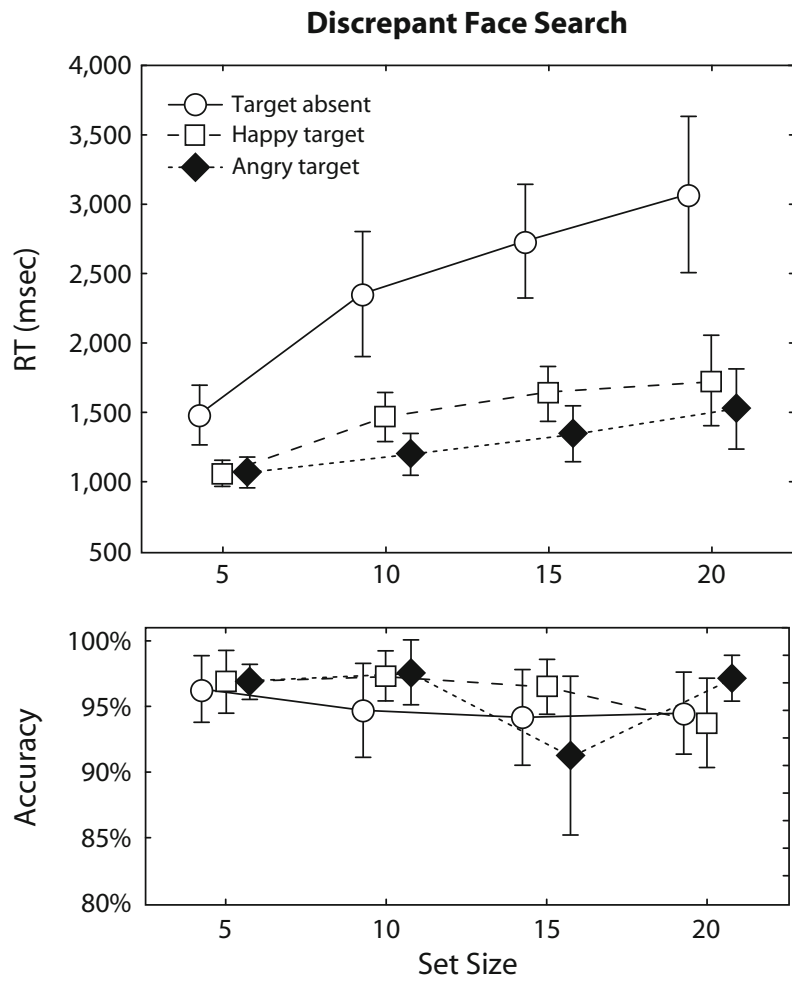

Figure 2. Mean response times and accuracies, with standard error bars for each of the conditions in Experiment 1.

\section{EXPERIMENT 2}

\section{Top-Down Facial Expression Search}

The objective of Experiment 2 was to investigate whether a top-down search goal for a facial expression (i.e., looking for only a happy face or only an angry face) would modify the angry face superiority effect. In addition, we investigated whether the mere presence of a happy or angry face in the display, in opposition to the task goal, would influence the visual search. In Experiment 2, the participants viewed a number of schematic faces on a computer screen and determined either the presence or absence of a specific facial expression. Three different display types were included: all neutral faces, one target present and the rest neutral, or one nontarget singleton present and the rest neutral.

We conducted RT distribution analyses to further evaluate the search process. We used the ex-Gaussian model (Ratcliff \& Murdock, 1976) to summarize the RT distributions. The ex-Gaussian is the convolution of a normal distribution and an exponential distribution, and provides summary estimates of RT distribution shape in terms of $\mu$ (reflecting the leading edge of the RT distributionthe quickest responses), $\sigma$ (reflecting the variability in the RT distribution), and the $\tau$ (capturing the positively skewed tail of the RT distribution, reflecting the slowest responses). The ex-Gaussian parameters were extracted using quantile maximum likelihood estimation methods (Brown \& Heathcote, 2003). RT distribution shape was used by Hockley and Corballis (1982) to evaluate search processes in the Sternberg paradigm. They found that the increase in mean RT as a function of set size was captured by a skewing of the tail of the RT distribution (consistent with a serial-terminating search) rather than being caused by an increase in the leading edge of the distribution (as would be expected with a serial-exhaustive search). Given the special status of an angry face to attract attention, we hypothesized that the search for angry faces would occur in an automatic, parallel fashion. Thus, we would expect the same pattern found by Hockley and Corballis to be found in the present study: $\mu$ would be constant across set sizes, and the mean RT increase would be captured by an increase in $\tau$ as set size increases.

\section{Method}

Participants. Eleven young adults (mean age $=23.1$ years, 7 females) participated in exchange for class credit.

Stimuli and Apparatus. The apparatus and stimuli were identical to those used in Experiment 1. Each display included 5, 10, 15, or 20 face stimuli.

Procedure. Each participant took part in two sessions. In one session, the participants indicated the presence or absence of a happy face. In the other session, the participants indicated the presence or absence of an angry face. The order of the sessions was counterbalanced over participants. Each session started with an instruction screen. When the participants were ready, they pressed the space bar to begin the trial. Each display remained on the screen until the participants pressed the K or F key to indicate either the presence or absence of the target face. After the participants responded by pressing a key, a blank screen appeared for $200 \mathrm{msec}$ before the next trial display. The key assignment was counterbalanced over participants. A total of 1,680 trials were included in this experiment. ${ }^{1}$ For each session, one third of the trials included all neutral faces. On another one third of the trials, the target face appeared (either happy or angry, depending on the goal of the session). For the remaining one third of the trials, a nontarget singleton appeared. For the singletonabsent trials, all of the faces were homogeneous, so in this condition, not only was the target absent, but the nontarget singleton was also absent. For the nontarget singleton-present condition, there was a singleton face present in the display, which was not a target.

\section{Results}

Mean RT and accuracy for each of the conditions are presented in Figure 3. The mean RT and accuracy data were submitted to three-way ANOVAs, with task (happy face search, angry face search), display type (target present, nontarget singleton present, singleton absent), and set size $(5,10,15,20)$ as within-subjects variables. For the RT analysis, significant main effects were observed for display type $\left[F(2,20)=20.0, p<.0001, \eta_{\mathrm{p}}^{2}=.67\right]$ and set size $\left[F(3,30)=108.5, p<.0001, \eta_{\mathrm{p}}^{2}=.92\right]$. These results indicated that RT was faster for the angry face search than for the happy face search, and RT increased as the set size increased. Significant two-way interactions were observed for task $\times$ display type $[F(2,20)=16.7$, $\left.p<.0001, \eta_{\mathrm{p}}^{2}=.63\right]$ and display $\times$ set size $[F(6,60)=$ $\left.20.8, p<.0001, \eta_{\mathrm{p}}^{2}=.68\right]$. The task $\times$ display type interaction showed that the target-present trials were faster than the nontarget singleton-present trials for the angry face search; however, for the happy face search, the gain of the target-present trials over the nontarget singletonpresent trials was smaller. The display $\times$ set size interaction indicated smaller search slopes for the target-present 

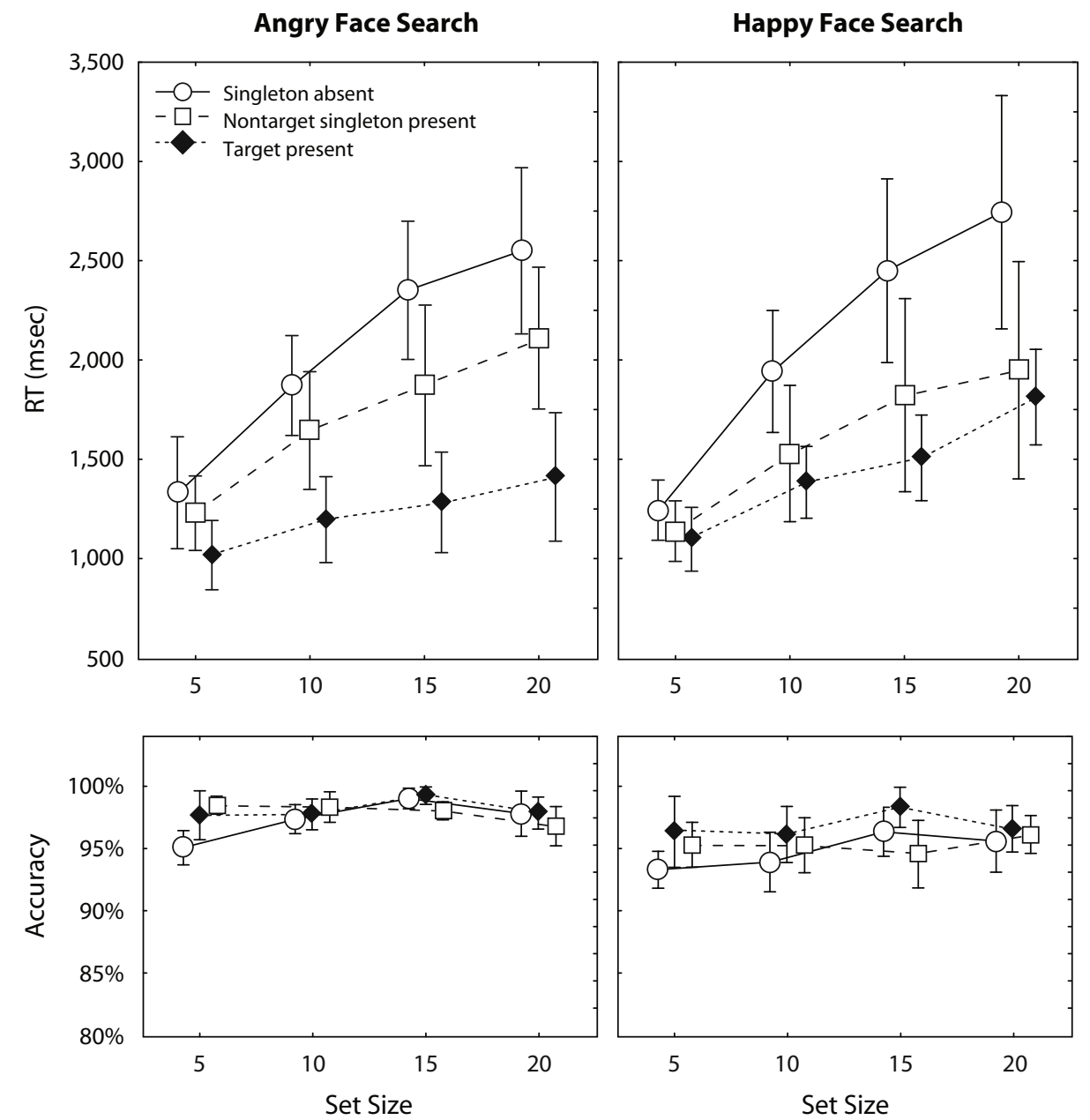

Figure 3. Mean response times and accuracies, with standard error bars for each of the conditions in Experiment 2.

trials in comparison with the nontarget singleton-present or singleton-absent trials.

A significant three-way interaction was observed for task $\times$ display type $\times$ set size $[F(6,60)=4.5, p<.001$, $\left.\eta_{\mathrm{p}}^{2}=.31\right]$, signifying that the search slopes for the angry face search and happy face search showed different patterns. The average search slopes for the angry face search were 28,59 , and $86 \mathrm{msec}$ for the target-present, nontarget singleton-present, and singleton-absent conditions, respectively. For the happy face search, average search slopes were 44, 57, and 104 msec for the target-present, nontarget singleton-present, and singleton-absent conditions. Post hoc analyses with Duncan's test $(\alpha=.05)$ revealed that the search slopes for the angry face search conditions were all different from each other, whereas search slopes for the happy face search target-present and nontarget singleton-present trials were equivalent, with the singleton-absent trial slope being larger. Additional post hoc analyses with Duncan's test $(\alpha=.05)$ indicated that for the target-present trials, the angry face search slope was larger than the happy face search slope, but for the nontarget singleton-present trials, the search slopes for the angry and happy face searches were equivalent. For the singleton-absent trials, the search slopes for the angry and happy face search did not show statistical differences.

For accuracy, significant main effects were observed for task $\left[F(1,10)=14.4, p<.005, \eta_{\mathrm{p}}^{2}=.59\right]$, display type $\left[F(2,20)=17.6, p<.0001, \eta_{\mathrm{p}}^{2}=.64\right]$, and set size $\left[F(3,30)=8.8, p<.001, \eta_{\mathrm{p}}^{2}=.92\right]$. The angry face search showed a higher accuracy than the happy face search, and target-present trials were more accurate than nontarget singleton-present trials or singleton-absent trials. A significant interaction was obtained for display $X$ set size $\left[F(6,60)=5.2, p<.001, \eta_{\mathrm{p}}^{2}=.68\right]$. The singleton-absent trials showed better accuracy with larger set size, whereas target-present and nontarget singleton-present trials showed constant accuracy over the increasing set size.

\section{RT Distribution Analyses}

Another indication of the nature of the search process depends on whether the RT distribution shifts up as the set size increases (i.e., the quickest responses are slowing) or whether the distribution becomes more skewed (i.e., the slowest responses are slowing). If the detection process is 
parallel (and its capacity is not exceeded), the quickest responses should be governed by this process, and $\mu$ should remain unchanged as set size increases. We used the quantile maximum likelihood estimation method (Brown \& Heathcote, 2003) to extract summary parameters from the RT distributions. We extracted parameter estimates for each participant and then averaged those parameter values. The average $\mu, \sigma$, and $\tau$ values are presented in Figure 4.

For the angry target-present trials, $\mu$ remained constant as a function of set size, which indicated that the quickest responses in the angry search were not influenced by set size (slope $=3 \mathrm{msec}$ ). Although not definitive (see Townsend \& Wenger, 2004), this finding is consistent with an underlying parallel search process governing angry face detection. For the happy target-present trials, $\mu$ increased with increasing set size (slope $=20 \mathrm{msec}$ ). The post hoc comparison with Duncan's test $(\alpha=.05)$ indicated that when the display included a target, $\mu$ slope was smaller for the angry face search than for the happy face search.

On the other hand, for the nontarget singleton-present conditions from the angry face search and happy face search, the average $\mu$ increased with increasing set size (34 msec and $33 \mathrm{msec}$ for the angry and happy nontarget singleton, respectively). Additional post hoc comparison showed that the $\mu$ slopes for the nontarget singletonpresent trials were equivalent for the happy face search and the angry face search. That is, the special status of the angry face was not observed in the nontarget singleton-
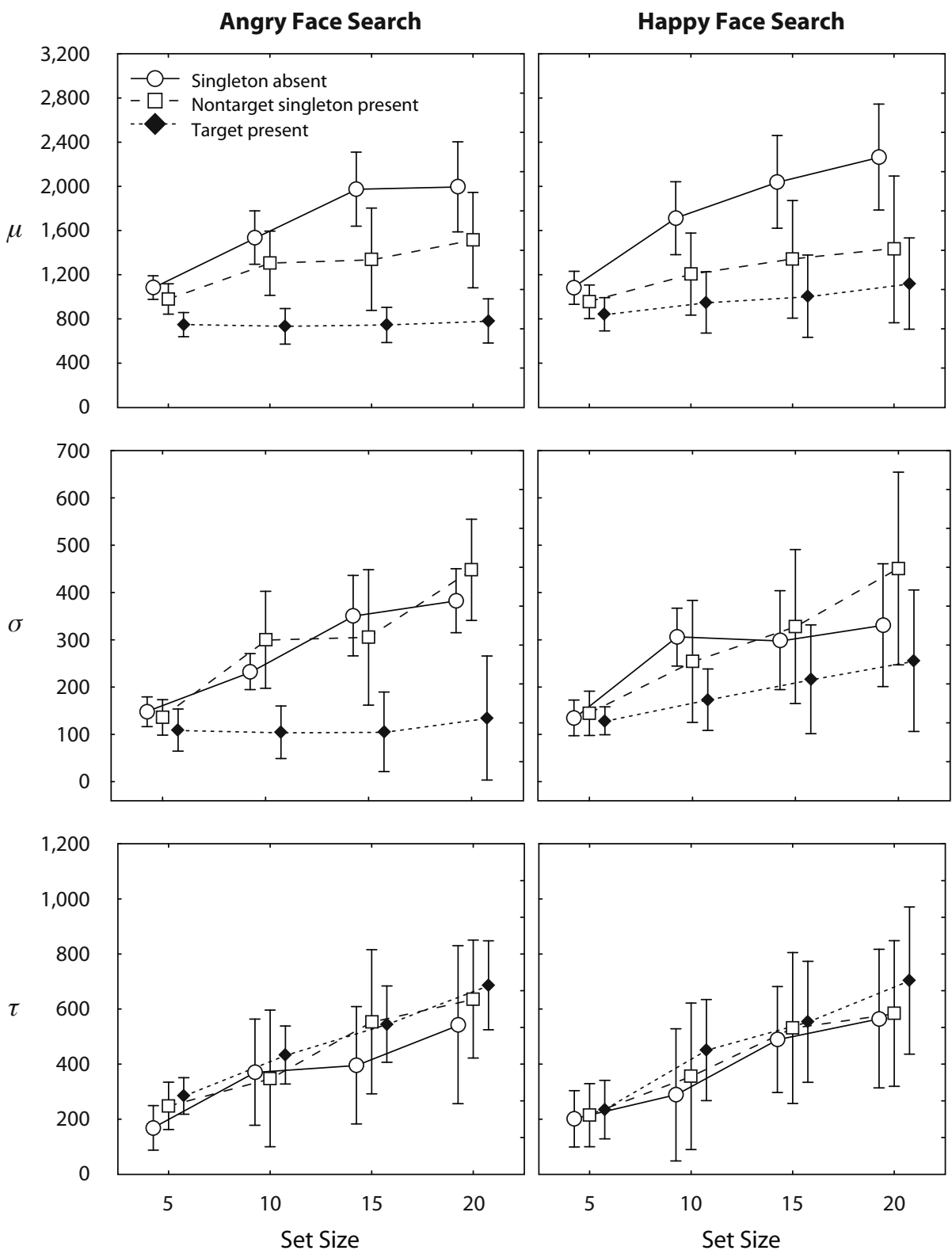

Figure 4. Mean values of the RT distribution parameters $\mu, \sigma$, and $\tau$ in Experiment 2. 
present condition. If the angry face superiority effect is based solely on a stimulus-based search process, the effect should be observed regardless of the top-down search goal.

The $\sigma$ values, which represent the variability of the RT distribution, showed a similar pattern as the $\mu$ values. For the target-present trials, the angry face search $\sigma$ remained constant over the increasing set sizes, whereas for the happy face search, $\sigma$ increased with increasing set sizes. In addition, $\sigma$ values for the singleton-absent trials and nontarget singleton-present trials showed similar slope over set size, suggesting that singleton-absent and nontarget singleton-present trials influenced RT variability in a similar way. This result also suggests that the mere existence of a nontarget singleton in opposition to the task goal does not support a special status for an angry face. The $\tau$ values increased over set size for both target-present and nontarget singleton-present conditions for both the angry and happy face search. This signaled that the slowest responses were slowing as set size increased.

\section{Discussion}

On the basis of the results of Experiment 2, we suggest that visual search efficiency is based on the combined influence of a top-down goal and stimulus characteristics with biological significance. When the display included a target, the angry face search RT slope was smaller $(28 \mathrm{msec})$ than the happy face search slope $(44 \mathrm{msec})$. Therefore, the angry face search appears to be more efficient than the happy face search when mediated via a top-down goal. However, when the angry or happy face was present in the display as a nontarget singleton, the two search slopes were equivalent (57 and $59 \mathrm{msec}$, respectively).

The RT distribution analysis showed distinctive patterns for the top-down angry and top-down happy face search. For the target-present trials, the angry face search showed constant $\mu$ over the increasing set sizes, indicating that fast responses were not influenced by set size, whereas the happy face search showed an increasing $\mu$ over set size. On the other hand, there was no difference in $\mu$ between the angry and happy nontarget singleton conditions. The $\sigma$ values supported that the nontarget singleton-present trials were processed more similarly to the singleton-absent trials. The RT distribution analyses complement the RT slope analyses and signal a special status for angry faces only during top-down search.

\section{GENERAL DISCUSSION}

We investigated how a top-down goal modified attentional bias for threatening facial expressions. In Experiment 1, participants searched for a discrepant facial expression in a homogeneous crowd of neutral faces. Consistent with previous research that has reported an angry face superiority effect, we obtained a smaller search slope when the target was angry than when it was happy. In Experiment 2, participants were instructed to search for a specific fa- cial expression (i.e., angry face or happy face). With the specific instruction of the target type, we attempted to observe a top-down, goal-driven search. The results of Experiment 2 supported the idea that even with a topdown goal, the angry face search slope was smaller than the happy face search slope, which supports the special status of an angry face to guide attention. However, when the happy or angry face was present in the display in opposition to the task goal (i.e., as a nontarget singleton), we obtained an equivalent search slope for both the angry and happy face search. RT distribution analyses also supported that a difference between the angry and happy face search was found only during the target-present trials (i.e., when guided by a top-down goal).

Visual search for threat faces takes place under the joint influence of a top-down goal and a stimulus with unique characteristics (i.e., a biologically relevant stimulus). For instance, neuropsychological studies have suggested that a quick and dirty amygdala circuit is responsible for the efficient detection of the threatening facial expressions. It is suggested that this amygdala circuit plays a critical role in automatic responses to a threat (LeDoux, 1996; Öhman, 2002). In addition, it has been shown that individuals with bilateral amygdala damage show impaired social judgment on the basis of facial expressions, especially those expressions involving threat-related emotions (Adolphs, Tranel, \& Damasio, 1998). In Experiment 1, the effectiveness of the angry face in guiding attention reflects attentional guidance based on stimulus characteristics (a high-priority stimulus). In Experiment 2, the top-down goal (as in happy or angry face search) guided visual search for facial expressions, and even with the topdown search goal, the angry face search was more effective than the happy face search. That is, when the target was present, the angry face search slope was smaller than the happy face search slope. However, when the angry or happy face singleton was present in the display in opposition to the task goal (as a nontarget singleton), the search slopes were equivalent for the happy and angry face, because both stimuli signaled (to the same degree) the termination of the search according to the top-down goal. Therefore, it appears that the special status of an angry face must be considered in conjunction with a top-down goal, even though the top-down goal did not override the stimulus-based search. Instead, the two types of guidance combine to influence visual search for threat faces.

The present results suggest that a top-down goal plays a key role during facial expression search. Neuropsychological studies have suggested an evolutionary account for the angry face superiority effect. That is, one's ability to detect a threat or danger effectively would be essential for survival of the species. However, a survival value of any function may depend on the goal at hand. For instance, excessive or constant vigilance to physical threat is not always a good survival strategy. The present study supports the idea that an alert to threat as well as a top-down goal combine to influence facial expression search. 


\section{AUTHOR NOTE}

Correspondence concerning this article should be addressed to S. Hahn, Department of Psychology, University of Oklahoma, 455 West Lindsey Street, Norman, OK 73019 (e-mail: sowon@ou.edu).

\section{REFERENCES}

Adolphs, R., Tranel, D., \& Damasio, A. R. (1998). The human amygdala in social judgment. Nature, 393, 470-474.

Brown, S., \& HeAthcote, A. (2003). QMLE: Fast, robust, and efficient estimation of distribution functions based on quantiles. Behavior Research Methods, Instruments, \& Computers, 35, 485-492.

Eastwood, J. D., Smilek, D., \& Merikle, P. M. (2001). Differential attentional guidance by unattended faces expressing positive and negative emotion. Perception \& Psychophysics, 63, 1004-1013.

Dimberg, U., Thunberg, M., \& Elmehed, K. (2000). Unconscious facial reactions to emotional facial expressions. Psychological Science, 11, 86-89.

Fox, E., Lester, V., Russo, R., Bowles, R. J., Pichler, A., \& Dutton, K. (2000). Facial expressions of emotion: Are angry faces detected more efficiently? Cognition \& Emotion, 14, 61-92.

Fox, E., Russo, R., Bowles, R., \& Dutton, K. (2001). Do threatening stimuli draw or hold visual attention in subclinical anxiety? Journal of Experimental Psychology: General, 130, 681-700.

Hahn, S., Carlson, C., Singer, S., \& Gronlund, S. D. (2006). Aging and visual search: Automatic and controlled attentional bias to threat faces. Acta Psychologica, 123, 312-336.

Hansen, C. H., \& Hansen, R. D. (1988). Finding the face in the crowd: An anger superiority effect. Journal of Personality \& Social Psychology, 54, 917-924.

HocKLEY, W. E., \& CoRballis, M. C. (1982). Tests of serial scanning in item recognition. Canadian Journal of Psychology, 36, 189-212.

LeDoux, J. E. (1996). The emotional brain: The mysterious underpinnings of emotional life. New York: Simon \& Schuster.
Mather, M., \& Carstensen, L. L. (2003). Aging and attentional biases for emotional faces. Psychological Science, 14, 409-415.

MogG, K., \& Bradley, B. P. (1999). Orienting of attention to threatening facial expressions presented under conditions of restricted awareness. Cognition \& Emotion, 13, 713-740.

Morris, J. S., Öhman, A., \& Dolan, R. J. (1998). Conscious and unconscious emotional learning in the human amygdala. Nature, 393, 467-470.

NothduRFT, H.-C. (1993). Faces and facial expressions do not pop out. Perception, 22, 1287-1298.

Öhman, A. (2002). Automaticity and the amygdala: Nonconscious responses to emotional faces. Current Directions in Psychological Science, 11, 62-66.

Öhman, A., Lundqvist, D., \& Esteves, F. (2001). The face in the crowd revisited: A threat advantage with schematic stimuli. Journal of Personality \& Social Psychology, 80, 381-396.

Ratcliff, R., \& Murdock, B. B., Jr. (1976). Retrieval processes in recognition memory. Psychological Review, 83, 190-214.

Townsend, J. T., \& Wenger, M. J. (2004). The serial-parallel dilemma: A case study in a linkage of theory and method. Psychonomic Bulletin \& Review, 11, 391-418

Vuilleumier, P., \& Schwartz, S. (2001). Emotional facial expressions capture attention. Neurology, 56, 153-158.

Williams, M. A., Moss, S. A., Bradshaw, J. L., \& Mattingley, J. B. (2005). Look at me, I'm smiling: Visual search for threatening and nonthreatening facial expressions. Visual Cognition, 12, 29-50.

\section{NOTE}

1. We included more trials in Experiment 2 than in Experiment 1 because the RT distribution analysis we employed requires a minimum of 40 trials per condition. We did not find any significant effects of block.

(Manuscript received August 25, 2005; revision accepted for publication May 5, 2006.) 